\title{
Symbol Characters Allocation of a QWERTY Type Keyboard Design for Smartphones
}

\author{
Kuk Kim* \\ Department of IE, Seokyeong University, Seoul, Korea
}

(Received: November 3, 2014 / Accepted: November 11, 2014)

\begin{abstract}
The QWERTY type keyboard is a classical device that has been used for computers for a long time. The keyboard design of mobile devices like smartphones is an important issue to consider because of the limited space on the touch screen. This paper presents a design for symbol allocation on the QWERTY type soft keyboard. A 27-cell model, including the full stop (.), is proposed in this paper. A QWERTY type keyboard for smartphones is mainly known for its spatial compatibility, whereas the characters of the ANSI keyboard are allocated to the shoulder positions for functional auxiliary input methods such as the long pressing method.
\end{abstract}

Keywords: Keyboards, Smartphones, QWERTY Type, ANSI, Symbol Allocation, Shoulder Position

* Corresponding Author, E-mail:kimkuk99@daum.net

\section{INTRODUCTION}

The QWERTY type keyboard has been the classical type of keyboard for both typewriters and computers for a long time. The layout shown in Figure 1 is the standard layout of the USA's visual arrangement of keys on a computer, or other typographic keyboard (ANSI keyboard, 1988).

Bemer (1982) studied the standards for keyboards for mental aspects beyond physical aspects considering human factors.

Currently, the touch screen keyboards of smartphones

\begin{tabular}{|c|c|c|c|c|c|c|c|c|c|c|c|c|c|c|c|}
\hline \begin{tabular}{|l|l|} 
& $!$ \\
\end{tabular} & $\begin{array}{l}@ \\
2 \\
\end{array}$ & & & $\begin{array}{l}\$ \\
4 \\
\end{array}$ & $\begin{array}{l}\% \\
5\end{array}$ & $\hat{6}$ & $\begin{array}{l}8 \\
7 \\
\end{array}$ & 8 & & & $\begin{array}{l}\text { ? } \\
0\end{array}$ & 1 & + & & Eckspace \\
\hline 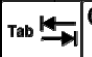 & $\mathbf{Q}$ & $\mathbf{w}$ & $\mathrm{E}$ & R & $\mathbf{T}$ & $\sqrt{1}$ & $\vec{Y}$ & $\mathrm{U}$ & 1 & 0 & F & & & & $\pi$ \\
\hline \begin{tabular}{|l|} 
Caps Lock \\
\$
\end{tabular} & $=\mathrm{A}$ & $\mathbf{S}$ & $\bar{D}$ & & $\vec{F}$ & $\overline{\mathbf{G}}$ & $\mathrm{H}$ & $\mathbf{J}$ & $K$ & L & L & : & ", & Enter & \\
\hline $\begin{array}{c}\text { Shift } \\
\text { Sิ }\end{array}$ & & Z & $\mathbf{x}$ & C & V & B & A & & $M$ & $<<$ & $>$ & & & & \\
\hline $\mathrm{Crr}$ & $\begin{array}{l}\text { Win } \\
\text { Key }\end{array}$ & Alt & & & & & & & & & Alt & & $\begin{array}{l}\text { Win } \\
\text { Key }\end{array}$ & Menu & Crir \\
\hline
\end{tabular}

Figure 1. ANSI keyboard layout used primarily in the United States. or smart devices are widely following the QWERTY computer keyboard layout, and similar to the QWERTY keyboard, the touch screen keyboard also consists of around 26 Latin letters. A good example of this is the keyboard layout of the iPhone as is shown in Figure 2. It is, however, important to note that the layout of the QWERTY type keyboard found on smartphones is somewhat different from the computer keyboard's layout because of limited space.

Many symbols (i.e., the 42 characters shown in Figure 1, such as the ten digits 0 to 9 , the comma, and so

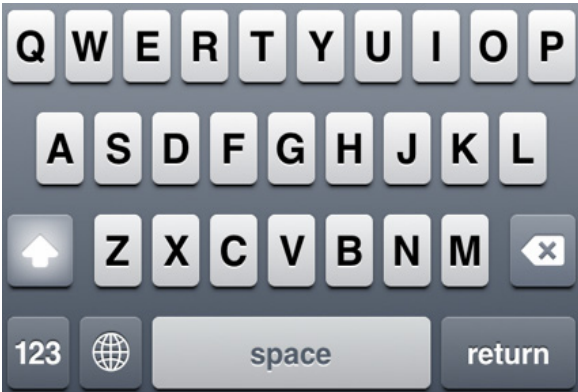

Figure 2. Keyboard layout of iPhones. 


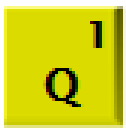

Figure 3. An example of dual assignment.

on) are not assigned directly and cannot be input by a single tapping for many smartphones. However, touch screens of smartphones have many functional input methods, such as long pressing. Therefore, other symbols can be assigned simultaneously with the 26 Latin letters shown here in Figure 3.

This paper proposes a character allocation design for touch screen keyboards of smartphones. The allocation of non-graphic keys and function keys, such as level 2 select (frequently called 'shift'), 'capitals lock,' 'return,' 'backspace,' etc., is not discussed in this paper although the arrangement of these function keys is another important issue. Neither the space bar will also be discussed here which is generally located between nongraphic function keys of the lowest row.

\section{PRELIMINARY AND ASSUMPTIONS}

This paper focuses on the design of three rows of graphic characters except for the space bar. There is no row for the digits 0 to 9 . A row for these digits will give an advantage of the direct input of digits, but this will result in a smaller display window on the screen. Therefore, the auxiliary method, for example, the long pressing method of a basic alphabet key is used to input digits in this paper.

The alphanumeric zone of the ISO/IEC 9995-2 (2009) includes the 83 invariant graphic characters of the 52 small and capital Latin letters, the digits 0 to 9, the character space, and 20 symbol characters as is shown in Table 1. However, the ANSI keyboard has an extra 12 symbols as is shown in Table 1.

\subsection{Symbol Allocation of Some Smartphones}

We have researched the symbol allocation of basic keyboards of some smartphones. As shown in Figure 2, iPhones have no symbols even though accent letters,

Table 1. Symbols arranged in the ANSI keyboard

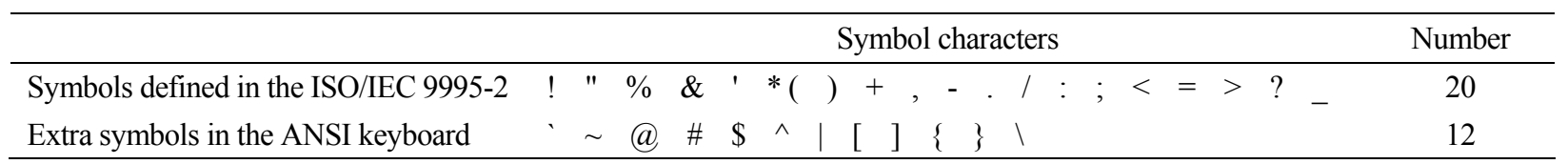

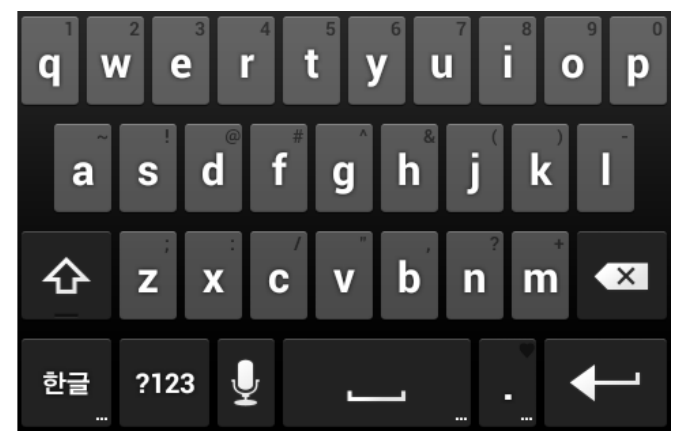

(a)

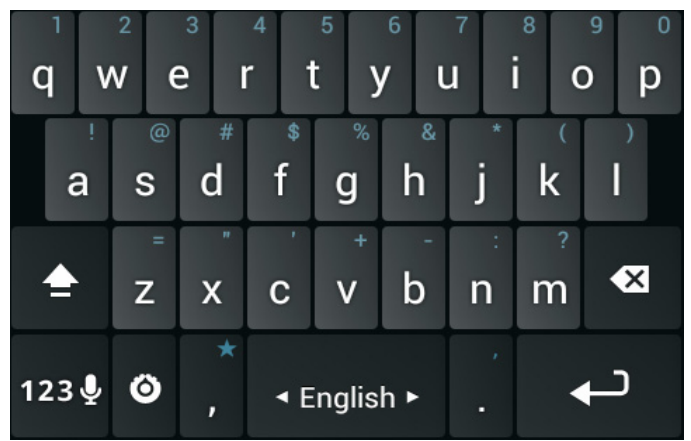

(c)

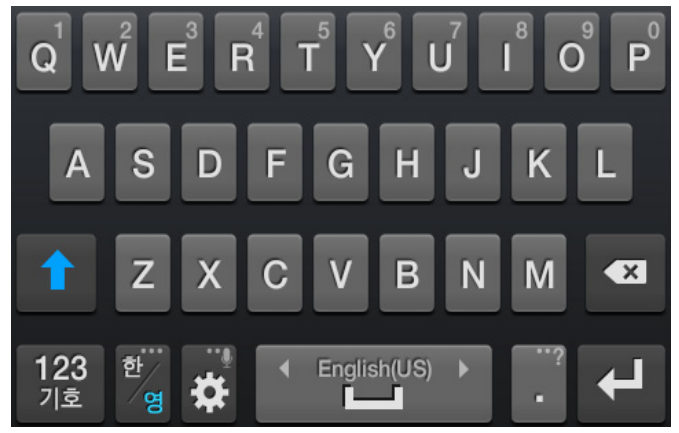

(b)

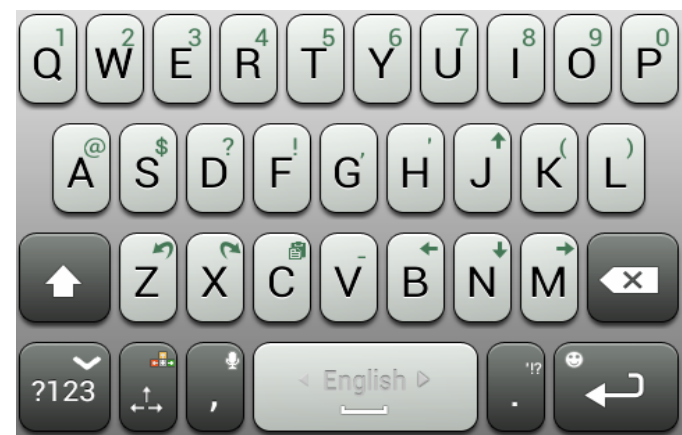

(d)

Figure 4. Some kinds of keyboards of smartphones. (a) GOOGLE keyboard, (b) SAMSUNG keyboard, (c) MULTILING keyboard, and (d) A.I.TYPE keyboard. 
such as À are possible by long pressing.

Figure 4 shows some kinds of keyboards, representatively, found on smartphones as well as Figure 2. There are many soft keyboards than these. Many soft keyboards allocate symbols on shoulder positions of keys, but specific positions are different respectively. The ten digits 0-9 can be found on the shoulder positions of the first row and are the same for all the keyboards. However, the second and the third row of the SAMSUNG keyboard, for an example, have no symbols when compared to the other keyboards, and so on.

In Figure 4, it is reasonable that, for example, hidden symbols, ' $[$, \{, and <' have similarity to '(' by the similar geometric shapes and the conceptual compatibility as 'open'. But, in the case of a group, for example, '!, $\boldsymbol{\&}$, \#, and \%', they have low compatibility each other.

\section{NUMBER OF CELLS FOR BASIC ASSIGNMENT}

For recommendations of key size, values for the center-to-center spacing of keys are generally around 0.75 inches (about $1.9 \mathrm{~cm}$ ) (Lewis et al., 1997). Therefore, over 10 columns are very difficult for the lengthwise direction of phones, but 10 columns are unavoidable. For small full QWERTY keyboards, text entry becomes more problematic because of the small size of the virtual keys, absence of tactile feedback, and occlusion of virtual keys by fingers (Kwon et al., 2009).

We consider two alternative designs; the 26-cell model and the 27-cell model, see Figure 5. For reference, Hwang et al. (2012) designed a 27-cell QWERTY keyboard layout to implement a mental spelling system.

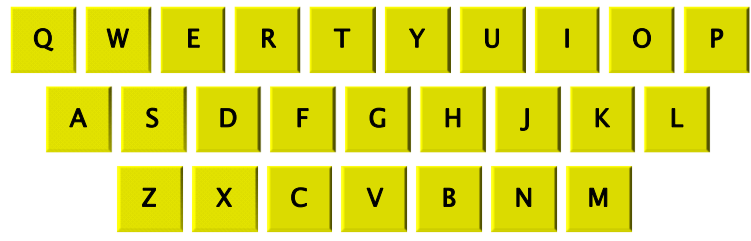

(a)

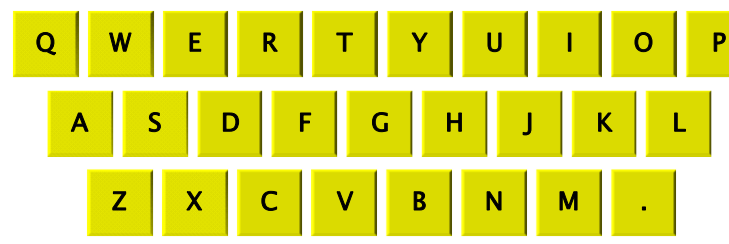

(b)

Figure 5. Two alternative designs: (a) the 26-cell model and (b) the 27-cell model.

The 27-cell model is preferred, because,

1) 10 cells for the 1 st row, 9 cells for the 2 nd row, and 8 cells for the 3rd row are a balanced design,

2) 1 extra cell can be used as the full stop punctuation (.), which is a frequently used punctuation in texts, and

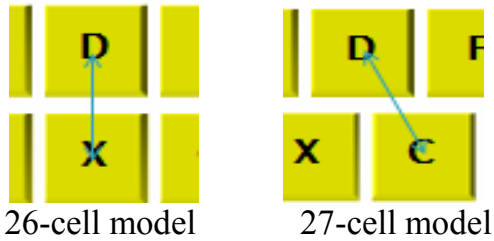

Figure 6. The center-to-center distance of the 2 nd and the 3rd row.

A survey for the two models, as shown in Figure 7, demonstrates that 30 of 32 students (age 19-23 years; 20 males and 12 females) preferred with the 27-cell model.

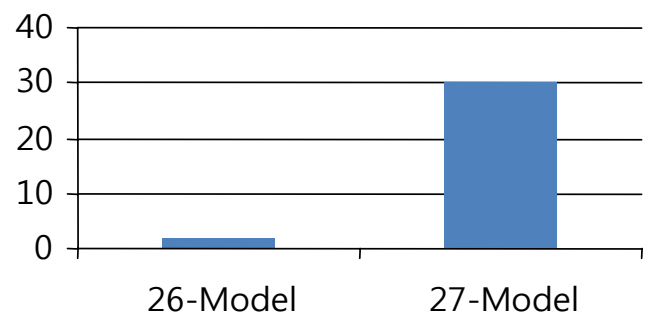

Figure 7. A survey for the two models.

3) The distance of the center-to-center spacing of the two adjacent keys of the 2 nd and the 3 rd rows is longer and more separable for the 27-cell model when compared to the 26-cell model (by the Pythagorean theorem). See the two arrows in Figure 6.

\section{ALLOCATION OF SYMBOL CHARAC- TERS}

There are many functional input methods for smartphones (for example, long pressing, long pressing and tap, long pressing and drag, drag, and so on). Therefore, extra symbol characters other than the alphabet letters can be used without switching keyboards simply by making use of dual arrangement. It is difficult to insert many symbol characters visually, so it is therefore suggested to insert a representative character at the shoulder position (see Figure 3).

\subsection{Ten Digit Characters}

The ten digits 0 to 9 are located in the first row as is shown in Figure 8. This allocation is due to the original location and the spatial compatibility (Williges et al., 1987) of computer keyboards. A survey about the allocation of the digits $0-9$ shows that 20 of 25 students (age 19-23 years; 16 males and 9 females; some participants are same of Figure 7) agree with this type of layout.

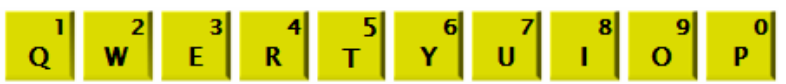

Figure 8. The first row. 


\subsection{Symbol Characters in the Second Row}

There are some alternative symbol allocations for the nine cells of the 2 nd row.

- Alternative A: To select nine symbols from ten shifted characters of the digits row of ANSI keyboard, [!], [@], [\#], [\$], [\%], [^], [\&], [*], [(], and [)], because of spatial compatibility.

- Alternative B: To select nine symbols from all symbols of ANSI keyboard (i.e., 32 symbols), depending on the importance of symbols.

Alternative $\mathrm{A}$ is considered more desirable, and a survey shows that 20 of 32 students (same participants of Figure 7) agree to this idea. There are nine keys in the second row, therefore, one symbol among ten symbols should be omitted. Which one is preferable? After a preliminary examination, these are some of the candidates;

[!] because it is a punctuation like the comma (,) and the remaining symbols have good spatial compatibility,

[\$] because it is a national currency symbol,

[^] because it is a accent symbol like ['] and ['], or others.

The result of the survey of 33 students (ages 19-23 years; 20 males and 13 females; 1 participant is added the survey of Figure 7) is shown in Figure 9.

Hence, nine symbols except the exclamation mark (!), are allocated in the 2nd row as is shown in Figure 10.

We grouped the 4 brackets as a group, ' $(,<,[,\{$ ' and the remaining 4 brackets as another group, '), $>$, l, \}', where '(' and ')' are the representative symbols due to their conceptual compatibility of shapes and the usage.

\subsection{Symbol Characters in the Third Row and Grouping}

The exclamation mark (!) is located in the first key of the 3 rd row. The number of remaining cells is 7 , and

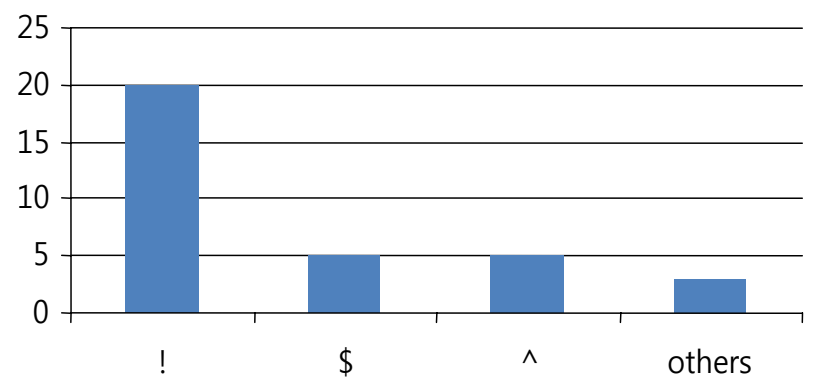

Figure 9. A symbol to omit in the 2nd row.

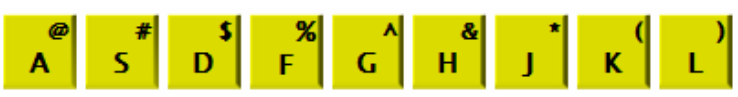

Figure 10. Symbol allocations in the 2nd row. the remaining symbols are $\left.15,\left[{ }^{\prime}\right],[\sim],[-],[]\right],[=],[+]$, $[\backslash],[]],[:],[;],['],["],[],,[/]$, and [?]. Seven symbols should be chosen among them.

This problem may be solved by two approaches. First, we need to consider the symbols in the basic positions on a computer keyboard (the set A in Figure 11), and the symbols of ISO 9995-2 (the set B in Figure 11). The common set of them are the 6 symbols, ['], [,], [-], [=], [/], and [;].

Second, we surveyed the user's preference about symbols. We requested to rank 1 to 5 among 15 symbols and the rank 1 scored 5 points, rank 2 scored 4 , and so on. We did not request to rank 1 to 15 , because it is difficult to rank 1 to 15 considering human's informationprocessing channel capacity (Knight, 1987).

Dell'Amico et al. (2009) studied the total frequency of usage for the single-finger keyboard layout problem. Here, only the space, the apostrophe, and the hyphen had significant frequencies. Total of 81 students (age 19-25 years; 46 males and 35 females) participated in my study. The result (sorted by descending scores) is as shown in Figure 12.

It is desirable to select the question mark (?) as one of 7 symbols, though it is not in the basic position on computer keyboards when compared with ['], [,], [-], [=], [/], and [;]. On the other hand, ["], ['], and [ ] are also ranked high. The quotation mark (") may be important, but ['] is ranked higher and is a basic positioning symbol on computer keyboards. Further, ["] can be obtained by doubling of [']. We think ['] may be confusing as a single quotation mark, and may be selected for psychological effect because of its first instance among 15 symbols. It has the geometric similarity or conceptual compatibility to the apostrophe ['].

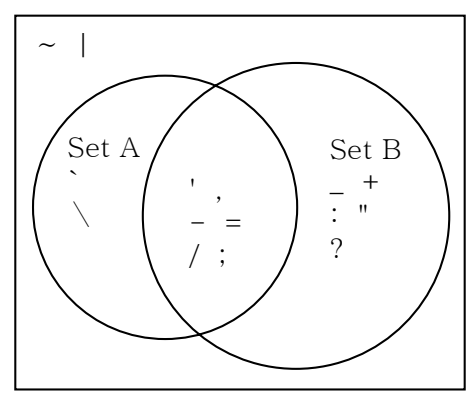

Figure 11. Classification of 15 symbols.

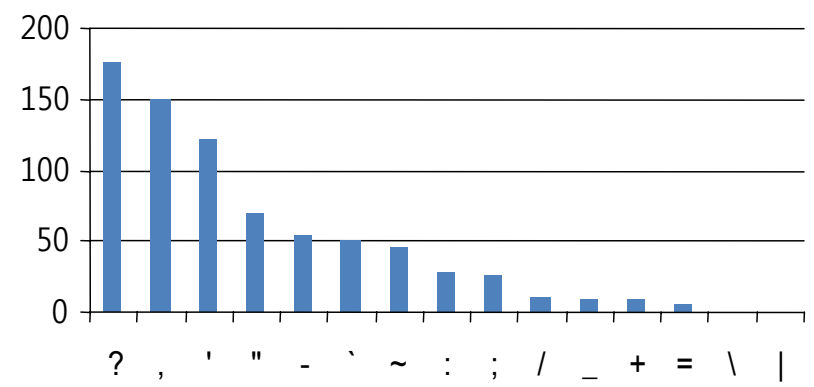

Figure 12. User's preference about symbols. 


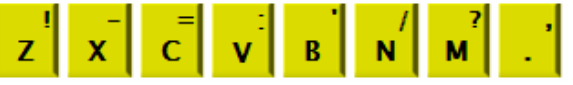

Figure 13. A design of the third row.

Though [ ] scored higher than $[/]$ and $[=]$, both of [/] and [=] are included in the 7 symbols due to its original location on a computer keyboard. It is difficult to decide what to choose between [;] and [:]. We chose [:] because we think it may be slightly more useful than [;], for example, 5:30 pm.

The end result is shown in Figure 13, where the specific location would follow the orders of a computer keyboard. The comma (,) is located in the full stop (.) key, and [?] is located near [.].

Now, we must group the remaining 8 symbols, [;], $\left[\mathrm{]},[+],["],\left[{ }^{\prime}\right],[\backslash],[\sim]\right.$, and [l] to the prior assigned 17 symbols by the compatibility or geometric similarity, a survey, and the ANSI keyboard's existing group such $\because, \begin{aligned} & + \\ & =\end{aligned}, \begin{aligned} & 1 \\ & 1\end{aligned}, \begin{aligned} & \vdots \\ & ;\end{aligned}, \begin{aligned} & ?, \\ & 1\end{aligned}$, and $\begin{aligned} & ? \\ & 1\end{aligned}$

According to a survey of 58 students (age 19-25 years; 33 males and 25 females) as shown in Figure 14, the 8 symbols are grouped to the already assigned 17 symbols as follows;

[;] is grouped in [:] for similar shape. The survey result shows the same.

[_] is grouped in [-] for similar shape. The survey result shows the same.

["] and ['] are in ['] because of its shape and usage as an accent symbol. The survey result shows the same.

$[\backslash]$ is in [/] for similar shape. The survey result shows the same.

The next three symbols are not easy to decide and we choose among alternatives. We consider [l] be allocated; 1) in [/] because it is located with [ $\backslash]$ on the computer keyboard and $[\backslash]$ is grouped to [/], or 2) in [!] because of its shape. The survey shows the first rank is [/] and the second rank is [!]. Therefore we decide [l] should be in [/].

We think alternatives for $[\sim]$ can be 1 ) in $[=]$ because it can be used as a 'similarity symbol' or, 2) in [-] because the usage is similar to that of the hyphen.

The survey shows the first rank is [-] and the second rank is [!]. The group of the second rank said they used $[\sim]$ and [!] as emotional symbols.

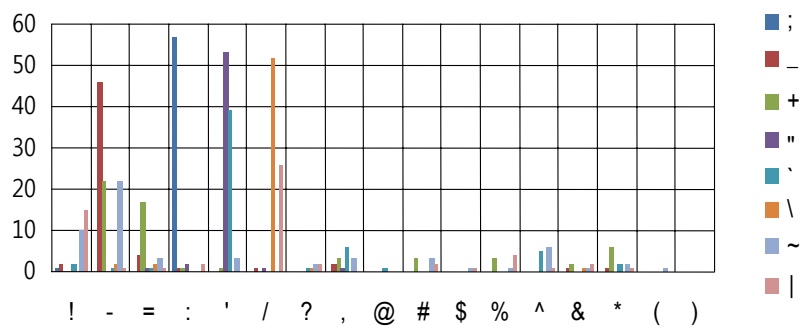

Figure 14. The compatibility of the 8 symbols to already assigned 17 symbols.

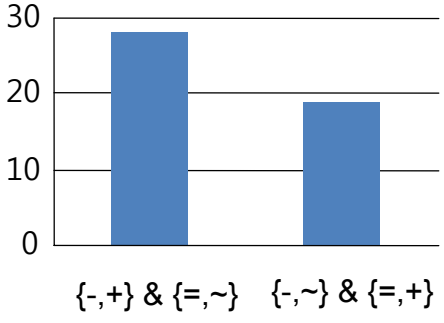

Figure 15. $[-]$ and $[=]$ versus $[+]$ and $[\sim]$.

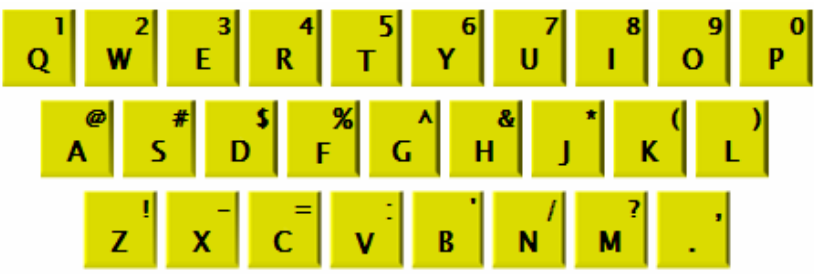

Figure 16. Dual arrangement of 27-cell model.

Table 2. Hidden symbols

\begin{tabular}{cc}
\hline Representative & Hidden symbol \\
\hline( & $<[\{$ \\
) & $>]\}$ \\
- & $+_{-}$ \\
$=$ & $\sim$ \\
$:$ & $;$ \\
1 & $" \checkmark$ \\
$/$ & $\backslash$ \\
\hline
\end{tabular}

We consider [+] be 1) in [-] because it is an arithmetic symbol, or 2) in [=] because of the position compatibility on the computer keyboard.

The survey shows the first rank is [-] and the second rank is $[=]$.

In order to adjust the number of symbols of [-] and [=] groups, we surveyed the compatibility of groups as Figure 15 by 47 students (ages 19-25; 25 males and 22 females). The Figure 15 shows grouping $\{-,+\}$ and $\{=$, $\sim$ is preferable.

\subsection{Result}

Figure 16 shows a dual arrangement of the 27-cell model with 27 symbols first at shoulder positions.

Hidden symbols are allocated as shown in Table 2.

\subsection{Other Symbols, For Examples}

There may be other extra symbols. Currency symbols, $[£],[€],[W]$, and so on, may be in [\$]. The standard keyboard of Great Britain have two more symbols such $\neg$ (not sign) and $£$ (pound sign) than ANSI. The 'not sign $(\neg)$ ' may be in [!] because [!] frequently means 'not' in computer programming. The broken bar ['] may be in [|] because of its shape. The centered dot (middle dot) may be in the [.] key. 


\section{CONCLUSIONS}

Symbols on shoulder positions of smartphones can be input by the auxiliary method, for example, the long pressing method. There may be other hidden symbols that have the compatibility to symbol characters of the visual shoulder characters.

We designed a 27-cell model for QWERTY type keyboard on smartphones, where the 27th cell is for the full stop mark. The digits and symbols on the computer keyboard are allocated and grouped to a smartphone keyboard as shown in Figure 16 and Table 2.

This paper may be useful to make an international standard by ISO/IEC JTC 1/SC 35 (User interfaces). Many non-graphic keys as Backspace, Shift, etc., were not considered in this paper, and this topic should be studied in a future.

\section{ACKNOWLEDGMENTS}

This Research was supported by Seokyeong University in 2012.

\section{REFERENCES}

ANSI keyboard layout (1988), http://en.wikipedia.org/ wiki/Keyboard_layout.

Bemer, R. W. (1982), Standards for keyboards, Сотриters and Standards, 1(2/3), 133-135.
Dell'Amico, M., Díaz, J. C. D., Iori, M., and Montanari, R. (2009), The single-finger keyboard layout problem, Computers and Operations Research, 36(11), 3002-3012.

Hwang, H. J., Lim, J. H., Jung, Y. J., Choi, H., Lee, S. W., and Im, C. H. (2012), Development of an SSVEPbased BCI spelling system adopting a QWERTYstyle LED keyboard, Journal of Neuroscience Methods, 208(1), 59-65.

ISO/IEC 9995-2 (2009), Information technology-Keyboard layouts for text and office systems-Part 2: Alphanumeric section.

Knight, J. L. (1987), Manual control and tracking, In: Salvendy, G. (ed.), Handbook of Human Factors, Wiley, New York, NY, 182-218.

Kwon, S., Lee, D., and Chung, M. K. (2009), Effect of key size and activation area on the performance of a regional error correction method in a touch-screen QWERTY keyboard, International Journal of Industrial Ergonomics, 39(5), 888-893.

Lewis, J. R., Potosnak, K. M., and Magyar, R. L. (1997), Keys and keyboards. In: Helander, M. (ed.), Handbook of Human-Computer Interaction, North-Holland, Amsterdam, 1285-1315.

Williges, R. C., Williges, B. H., and Elkerton, J. (1987), Software interface design, In: Salvendy, G. (ed.), Handbook of Human Factors, Wiley, New York, NY, 1416-1449. 